Revista Interdisciplinaria de Humanidades, Educación, Ciencia y Tecnología

Año VII. Vol. VII. N³3. Edición Especial III. 2021

Hecho el depósito de ley: pp201602FA4721

ISSN-L: 2542-3029; ISSN: 2610-802X

Universidad Nacional Experimental Francisco de Miranda (UNEFM). Santa Ana de Coro. Venezuela

Daniel Patricio Castro-Salinas; Sergio Constantino Ochoa-Encalada

DOI $10.35381 / \mathrm{cm} . \mathrm{v} 7 \mathrm{i} 3.579$

\title{
Gamificación en el proceso de interaprendizaje: Una experiencia en biología con Genially
}

Gamification in the process of interlearing: An experience in biology with Genially

\author{
Daniel Patricio Castro-Salinas \\ dpcastros06@est.ucacue.edu.ec \\ Universidad Católica de Cuenca, Azogues \\ Ecuador \\ https://orcid.org/0000-0002-6955-6221 \\ Sergio Constantino Ochoa-Encalada \\ scochoae@ucacue.edu.ec \\ Universidad Católica de Cuenca, Cuenca \\ Ecuador \\ https://orcid.org/0000-0003-3067-3719
}

Recibido: 15 de agosto de 2021

Aprobado: 15 de noviembre de 2021 


\title{
RESUMEN
}

La investigación tuvo como objetivo determinar la efectividad de la aplicación de Genially con alumnos de bachillerato de la Unidad Educativa "Carlos Lenin Ávila" en el asignatura de biología, mediante una investigación de tipo experimental considerando para el efecto la aplicación de un pretest y un postest, datos que fueron analizados mediante la estimación de medias aritméticas de los promedios obtenidos por los estudiantes en las evaluaciones con el uso de SPSS evidenciando un incremento en los promedios, lo cual demuestra los cambios evidentes en la consolidación de conocimientos a través de presentaciones interactivas y evaluaciones mediante Gamificación con lo que se concluye que la aplicación de la herramienta digital Genially en la transmisión de conocimientos científicos mejora el rendimiento académico de forma significativa.

Descriptores: Juego educativo; material didáctico; programa informático didáctico; enseñanza asistida por ordenador. (Palabras tomadas del Tesauro UNESCO).

\begin{abstract}
The objective of the research was to determine the effectiveness of the application of Genially with high school students of the Educational Unit "Carlos Lenin Ávila" in the subject of biology, through an experimental investigation considering for the effect the application of a pretest and a posttest, data that were analyzed by estimating the arithmetic means of the averages obtained by the students in the evaluations with the use of SPSS, showing an increase in the averages, which shows the evident changes in the consolidation of knowledge through interactive presentations and Gamification evaluations, which conclude that the application of the Genially digital tool in the transmission of scientific knowledge significantly improves academic performance.
\end{abstract}

Descriptors: Educational game; teaching materials; educational computer program; computer-assisted teaching. (Words taken from the UNESCO Thesaurus). 


\section{INTRODUCCIÓN}

Desde los aportes de Serna-Huesca et al. (2015), se establece que la realidad mundial es muy inestable y los cambios que se presentan diariamente son variados haciendo necesaria la adaptación de la sociedad a los hechos actuales en todos los ámbitos incluida la educación que con el avance de la ciencia y la tecnología ha desarrollado nuevas formas de socialización y enseñanza con cambios a los cuales los docentes deben adaptarse en un mundo globalizado en concordancia con el reporte de La Organización de las Naciones Unidas para la Educación, la Ciencia y la Cultura [UNESCO] (2017) que hace referencia a que para el año 2030 América latina y el Caribe cumpla con los planes y objetivos del E2030.

Cierto es que la globalización como sostiene Sierra-Villamil (2016) exige cambios y transformaciones profundas en todos los niveles de educación haciendo necesaria la innovación en todo ámbito de enseñanza, para Byrd-Orozco (2015) además se necesitan actores educativos comprometidos en el cambio no solo en la parte disciplinar de las asignaturas contempladas en los currículos de cada país sino también de forma interdisciplinar, multidisciplinar y transdisciplinar para enseñar, dejando de lado practicas tradicionalistas como la que el educador es el dueño de la verdad y que hay una sola forma de transmitir conocimientos desde los docentes a los alumnos.

De acuerdo con la legislación nacional considerando a la Constitución Nacional vigente creada por la Asamblea Nacional Constituyente (2008) quien sostiene que la educación es un eje fundamental en el desarrollo del país es así que en el artículo 27 estipula que la misma tiene que ser de calidad y generar los conocimientos y capacidades necesarias para una vez culminados los niveles de educación obligatoria los ciudadanos puedan crear y laborar, siguiendo en esta línea la Ley Orgánica de Educación [LOEI] del Ministerio de Educación del Ecuador [MINEDUC] (2011) en el artículo 2 en el literal "u" y el articulo 6 hacen referencia a que la educación será de calidad e innovadora, aspectos considerados en el Currículo nacional del MINEDUC (2016) en el país como valores fundamentales en el perfil de salida del bachillerato ecuatoriano. 
Además, según la Organización de la Naciones Unidas [ONU] (2020) con la pandemia más del $90 \%$ de los estudiantes a nivel mundial se han visto afectados por el cierre de los centros educativos decretados a causa de la pandemia, es así que Mendoza-Castillo (2020); García-Aretio (2020); Covarrubias-Hernández (2021) evidencian en sus trabajos como las afectaciones en esta calamidad demostraron que los docentes, estudiantes y padres de familia no estaban preparados para afrontar la realidad que se vive, debido a causas relacionadas principalmente a la falta de preparación de los educadores en innovación educativa y de la desigualdad en el acceso a internet y medios tecnológicos de las familias lo que ha acrecentado la brecha de aprendizaje.

Es así que Hermosa del Vasto (2015) considera a la innovación en educación como fundamental en el proceso de enseñanza aprendizaje de los alumnos para que puedan hacer frente a la convivencia en una sociedad globalizada haciendo uso de las Tecnologías de la Información y la Comunicación (TIC) lo que permitirá crear inteligencias múltiples que parte como mencionan Hepp-K et al. (2015) desde la formación de los docentes en el manejo de recursos tecnológicos y la necesidad de contar con los equipamientos necesarios en las escuelas y colegios de medios de tecnología necesarios para la transmisión de conocimientos (Arias-Gallegos, 2015).

Por otra parte, la carta magna elaborada por la Asamblea Nacional Constituyente (2008) en sus artículos 28 y 344 establece al nivel de bachillerato como obligatorio que deberá estar articulado a la educación superior, en relación al artículo 43 de la LOEI del MINEDUC (2011) que garantiza en 3 años de bachillerato obligatorio la preparación del o la ciudadana en proyectos de vida con las competencias y habilidades necesarias para su incursión a la sociedad y la posibilidad de acceso a la educación superior mediante la aprobación de un de un examen de grado como está estipulado en el artículo 198, literal uno inciso tres del reglamento de la Ley Orgánica de Educación Intercultural [RLOEI] (2015) lo que les permitirá obtener el grado de bachiller.

Además, el currículo nacional vigente desde el año (2016) contempla a las ciencias naturales como un área esencial en la formación del estudiante y dentro de ella a la 
biología como una de las asignaturas básicas en el proceso de interaprendizaje del alumno en Bachillerato General Unificado (BGU) en Ciencias Básicas con destrezas a ser cumplidas durante su formación que posteriormente serán evaluadas como parte del proceso obligatorio para la obtención del título de bachiller y por ende en la evaluación para el ingreso a la educación superior.

Es por eso que analizando los resultados del examen ser bachiller del año lectivo 2018 2019 del informe del Instituto Nacional de Evaluación Educativa [INEVAL] (2019)en el cual se evidencia que en el área de ciencias naturales los resultados obtenidos por los alumnos del tercer año de Bachillerato General Unificado de la Unidad Educativa "Carlos Lenin Ávila" de la parroquia Bayas, en el cantón Azogues de manera global alcanzan un nivel mínimo de conocimientos en el área es por tanto que se propone la búsqueda de estrategias innovadoras que permitan mejorar la forma de impartir los conocimientos hacia los estudiantes con el fin de mejorar su rendimiento en la evaluaciones que posteriormente deberán rendir como parte de su formación.

Por lo tanto, en el presente trabajo busca determinar la efectividad del uso de Genially en el proceso de enseñanza aprendizaje en el área de ciencias naturales específicamente en la asignatura de biología con estudiantes de bachillerato, lo que permitirá demostrar mediante experimentación la factibilidad o no de la implementación de estrategias innovadoras que usen recursos audiovisuales, textos interactivos y estrategias de Gamificación durante el proceso de interaprendizaje.

En la elaboración del apartado se han considerado artículos científicos obtenidos de bases de datos Redalyc, Scielo, Scopus, ProQuest y Dialnet con un periodo de tiempo entre los años 2015 y 2021 tanto a nivel mundial, regional y nacional, usando criterios de búsqueda como "educación" "Gamificación"; "Educación and Gamificación", "estrategias de Gamificación en Educación"; "educación and ciencias naturales" y teniendo en cuenta procesos de revisión bibliográfica.

Comenzaremos analizando lo que mencionan Villalustre-Martínez y Del Moral-Pérez (2015) en España quienes mencionan que la Gamificación cobra importancia en el 
proceso de enseñanza aprendizaje porque permite hacer frente a situaciones complejas y de difícil explicación y comprensión para las dos partes a través de juegos con reglas, retos y etapas a ir superando por el alumno para ir adquiriendo conocimientos que perduraran en el tiempo, para el caso hicieron uso de una presentación multimedia similar al videojuego "Los Sims" concluyendo que el juego despertó el interés y la motivación de los participantes por aprender a medida que los niveles de dificultad del juego se iban superando.

Por otra parte Nikoletta-Zampeta et al. (2020) en Finlandia y Grecia explican la importancia de la aplicación de estrategias de Gamificación en la enseñanza de estadística que comúnmente es considerada como una ciencia básica para el manejo de inversiones y negocios por lo que es necesaria la motivación constante al estudiante para que pueda aprender de forma tal que pueda resolver los problemas a enfrentar en el ámbito educativo y laborar usando la Gamificación basada en desafíos especialmente, demostrando que al usar estrategias de Gamificación el nivel de aprendizaje de estadística de los alumnos mejoró.

En el trabajo realizado por Real-Pérez et al. (2021) en España determinaron que la motivación tiene mucha importancia al momento de impartir clases a los alumnos ya que les permite expresar de mejor forma sus ideas, pensamientos y mejora la relación tanto intrínseca como extrínseca, motivación que fue conseguida mediante la aplicación de estrategias de Gamificación con un sistema de recompensas en el área de educación física permitiendo al estudiante tener más autonomía, seguridad y motivación al momento de la toma de decisiones facultando observar mejoraras notables tanto en el rendimiento individual como grupal del educando.

De acuerdo con Palazón-Herrera (2015) mediante una investigación cuantitativa, no experimental en base a una encuesta realizada en Venezuela y haciendo uso de las herramientas y recursos digitales Moodle, ClassBadges y YouTube estableció que la Gamificación usando el método de recompensas mediante insignias digitales provoca en el alumnado una sana competencia debido a que se colocan metas las cuales deben ser 
superadas, despierta el interés por aprender cosas nuevas ya que consideran que son evaluados de una manera más justa, se ven motivados constantemente a superarse a sí mismo y se esfuerzan por superar a los demás.

Continuaremos mencionando el trabajo realizado en el ámbito de Gamificación como estrategia activa dentro de las aulas de educación citando a Zepeda-Hernández et al. (2016) en México quienes en dos fases de investigación basadas en el aprendizaje, la identificación de actividades, la dinámica en clase y la forma de evaluación establecieron que los estudiantes al ser evaluados mediante Gamificación de manera constante reducen la tensión que se enfrentarían al ser sometidos a una sola evaluación final, se vuelven más solidarios con sus similares buscando la forma de ayudar a todos a superar los retos a los que se enfrentan en el juego y además mejoran la relación con sus docentes.

Finalmente, en el ámbito regional se analizó a Ortiz-Colón et al. (2018) en Brasil, autores que usando una metodología cualitativa a través de revisión teórica analizaron publicaciones académicas de bases de datos internacionales a cerca de los beneficios de la aplicación de Gamificación en la educación en un periodo comprendido entre los años 2011 y 2015, considerando 37 experiencias con resultados beneficiosos en la aplicación de la estrategia , la motivación por aprender y la incorporación del "juego" en el proceso de enseñanza aprendizaje lo que posibilita al alumno ser el creador de su conocimiento a través de la dedicación.

En el ámbito local Llerena-Medina y Rodríguez-Hurtado (2017) haciendo uso de aplicaciones en el Sistema Interactivo de Respuesta (IRS) para el caso específico el uso de Kahoot como herramienta de Gamificación en la enseñanza del idioma inglés en educación superior mediante un diseño cuasi experimental de aplicación a estudiantes que en su totalidad poseían un teléfono celular inteligente para ser partícipes del mismo a quienes se aplicó un post test que en comparación a un pre test aplicado anteriormente evidenciaron un incremento en la comprensión del vocabulario estas herramientas e- 
learning generan positividad y una mayor participación en las actividades a desarrollar en el aula añadiendo diversión.

Continuando con esta temática Macías-Espinales (2018) en Ecuador aplicando la plataforma Rezzly como estrategia de Gamificación con estudiantes de educación secundaria con edades comprendidas entre los 15 y 16 años en el área de matemáticas y haciendo uso de una metodología mixta con pruebas pre y post test que con la aplicación de la prueba t-Student corroboró una variación entre las dos pruebas concluyendo que la estrategia aplicada en la mejoró el rendimiento académico, el dominio de la competencia y la comprensión de la teoría de la asignatura de la de los estudiantes y adicional a esto la motivación también se vio incrementada.

Finalmente se considera en experiencias previas de uso de aplicaciones como herramientas de Gamificación en la educación a Holguín-García et al. (2020) en Ecuador quienes a través de una revisión sistemática evaluaron experiencias de varias investigaciones que han usado esta herramienta como estrategia de enseñanza en matemáticas encontrando que existen ocho artículos dentro de la temática analizada evidenciando que en todos consideran a la Gamificación como motivadora y que incrementa el rendimiento académico y fortalece el proceso de enseñanza - aprendizaje a través de la evaluación contante y la recompensa mediante insignias, puntos o posición en tableros.

Es por eso que se analiza las metodologías activas que como indican Silva-Quiroz y Maturana-Castillo (2017) son aquellas que centran sus métodos, técnicas y estrategias en la ejecución de actividades planteadas por el docente que permiten al estudiante participar de forma directa en la adquisición de nuevos conocimientos con un compromiso constante por parte de todos los actores educativos, consta de procesos planificados en los cuales el alumno desarrolla la participación, creatividad y reflexión sobre las tareas enviadas en tanto el docente cumple con un rol de mediador ya que las metodologías activas basan el proceso de enseñanza - aprendizaje en actividades más no en 
contenidos lo que faculta además ir evaluando constantemente y retroalimentando en caso de ser necesario.

Según Espejo y Sarmiento (2017) en el manual de apoyo docente de la Universidad Central de Chile se consideran como metodologías activas a las siguientes:

\section{Tabla 1.}

Principales metodologías activas usadas en educación.

\begin{tabular}{ll}
\hline Principales metodologías activas & Análisis de casos \\
& Aprendizaje basado en procesos - ABP \\
& Aula invertida o Flipped Classroom \\
& Aprendizaje basado en procesos \\
& Aprendizaje basado en procesos - Team Learning \\
Aprendizaje y servicio $-\mathrm{A}+\mathrm{S}$ \\
\hline
\end{tabular}

Fuente: Aportes del manual de apoyo docente de la Universidad Central de Chile

Metodologías que asociadas a las Tecnologías de la Información y la Comunicación (TIC) posibilitan la Gamificación de la educación en las aulas facilitando al docente dar seguimiento, apoyo y tutela a sus dirigidos a medida que los estudiantes van ampliando su propio aprendizaje que como ya se ha mencionado estará basado en las actividades que vayan desarrollando.

La Gamificación término que para Rodríguez y Campión (2015) consiste en la estrategia que posibilita asociar técnicas de juegos en la enseñanza o promoción de productos y servicios basada principalmente en la estrategia de recompensas digitales obtenidas a base de objetivos propuestos, estrategias y metas establecidas por parte del usuario en búsqueda de superar las dificultades que se vayan presentando en el proceso; aunque no necesariamente la Gamificación está ligada al uso de aparatos tecnológicos.

Para Contreras-Espinosa (2016) la Gamificación aplicada en la educación se presenta como una alternativa viable a aplicar dentro de las aulas debido a la versatilidad que 
presenta, la accesibilidad a la que se tienen en la actualidad con el desarrollo de la tecnología y los dispositivos móviles y está acorde a la realidad vivencial diaria en niños y adolescentes, se basada en la naturaleza propia del ser humano de ser competitivo y en la necesidad innata por aprender a superar problemas desde los más sencillos a los más complejos, similar situación que se afronta en un juego gamificado que al ir superando niveles los estudiantes van adquiriendo aprendizajes significativos.

La Gamificación al ser un proceso en la enseñanza considera actividades previamente planificadas, para Sánchez-Peris (2015) la planificación debe tener claro los objetivos a conseguir, las reglas que se van a seguir, los roles a cumplir por cada participante y la forma en la cual se van a ir evaluando los avances y el producto final, debe ser previamente socializada con los estudiantes y estar ajustada a la realidad vivencial y construida de forma participativa yendo desde experiencias personales a una realidad grupal, además debe ser adaptable a todas las fases del proceso de interaprendizaje. Continuando en esta línea la Gamificación para García-Lázaro (2019) separa lo lúdico de la enseñanza de conocimientos, hipótesis y operaciones mediante "juegos" de interacción que posibilitan mantener el interés del estudiante en la búsqueda de nuevos conocimientos superando los retos que se presentan diferenciándose así de los juegos de aprendizaje que si bien es cierto las dos estrategias hacen uso de elementos parecidos y cuentan con un sistema de distinciones similares la Gamificación mediante motivación constante e inspiración consigue aprendizajes significativos evidenciados en los resultados que obtienen los alumnos luego de aplicar esta técnica.

Según Beiliková (2019) al aplicar la Gamificación dentro de las aulas de clase podemos tener ventajas en comparación con los métodos tradicionales de enseña dentro de ellas considera a la motivación y al protagonismo del estudiantado como las primordiales, regula los procesos de enseñanza adaptándose al ritmo de trabajo de cada uno de los alumnos, desarrolla la creatividad, participación y compañerismo, reduce efectos negativos al afrontarse a errores que podrían presentarse ya que son considerados como 
parte el proceso, permite al docente dar acompañamiento y retroalimentación durante el desarrollo de actividades además de ser divertida y estar en constante innovación.

En educación la motivación es un factor importante a considerar ya que como mencionan Valenzuela et. al. (2015) es lo que impulsa al alumno a realizar las tareas propuestas por el docente mediante la activación de recursos cognitivos y motivacionales para aprender, motivación que para Ospina-Rodríguez (2006) puede ser "intrínseca y extrínseca" definida a su vez por "criterios internos o externos" concluyendo con Sellan-Naula (2017) quien resalta que la importancia de este aspecto radica en la importancia de tener alumnos motivados para el cumplimiento de metas individuales de forma voluntaria que generará nuevos conocimientos aplicables en el sociedad.

Por otra parte, según un reportaje del Diario Córdova S.A.U. (2015) la herramienta digital Genial.ly surge se la idea de un equipo multidisciplinar con el principal objetivo de permitir la creación de contenidos digitales interactivos en búsqueda de la mejora de presentaciones, en la educación, en el marketing y la difusión de contenido, de fácil manejo, versátil, con la posibilidad de un almacenamiento directamente en la nube para ir acumulando o cambiando contenidos de manera gratuita en su versión más básica con la opción de adquirir las versiones pro, master y team con mayores ventajas entre ellas la Gamificación y presentaciones interactivas, además según Hart (2021) se encuentra dentro de las principales herramientas usadas a nivel mundial para aprender.

Para Catalán y Pérez (2020) las ventajas se deben a la variedad de opciones que Genially presenta en comparación con otras herramientas tradicionales creadas para la difusión de contenidos debido a que cuenta con plantillas propias y modificables de la aplicación el interfaz de fácil interacción y compresión por parte de los usuarios, tiene la facilidad para adaptarse a las necesidades de los beneficiarios, constantemente está en actualización de contenidos y diseños, es compatible con ordenadores y teléfonos móviles que cuenten con acceso a Internet, cuenta con almacenamiento directo en la nube para cada usuario y posibilita el trabajo en equipo de forma colaborativa. 


\section{METODOLOGÍA}

Para el caso se planteó una investigación de tipo explicativa con un enfoque cuantitativo que como explican Hernández-Sampieri y Mendoza-Torres (2018), es el más apropiado cuando se quieren evaluar magnitudes, estimar la ocurrencia o no de fenómenos y demostrar o negar hipótesis ya que es más objetiva con patrones establecidos y prediseñados que facilita unificar resultados siguiendo proceso determinados a través de la lógica y el razonamiento deductivo permitiendo hacer inferencias desde lo muestral a lo global con datos lo más cercanos a la realidad.

De igual manera se consideró un alcance descriptivo - exploratorio que como indica Ramos-Galarza (2020) la investigación exploratoria posibilita indagar características que pueden ser analizadas desde lo cuantitativo con análisis de datos básicos permitiendo en análisis estadístico de los mismos, con un diseño de investigación de tipo longitudinal o evolutiva que como exponen Hernández-Sampieri et al. (2014) es la que permite recolectar datos en tiempos diferentes que posibilitan formular inferencias con respecto al cambio que se dio el por qué se dio y las consecuencias de estas modificaciones.

De igual manera para Pulido-Polo (2015) las técnicas para la recolección de datos según posibilitan organizar y analizar los datos que serán usados en la observación de la situación actual y el estado final del objeto de estudio por lo que para la presente investigación se han propuesto las siguientes técnicas e instrumentos de investigación.

Se consideró para el estudio a los alumnos del segundo año de Bachillerato General Unificado de la Unidad Educativa "Carlos Lenin Ávila" de la cuidad de Azogues - Ecuador que para el año lectivo 2021 - 2022 cuenta con una población de 13 estudiantes a los cuales se aplicó dos cuestionarios, un pretest con el objetivo de identificar los conocimientos adquiridos mediante una clase tradicional con el uso de diapositivas en Power Point y un postest con el uso de la herramienta digital Genially.

Los test se realizaron a través de Microsoft Forms y fueron aplicados de manera virtual considerando la modalidad de estudio vigente en la institución los mismos que se 
validaron con un Alfa de Cronbach de 0,87 y los datos recopilados fueron analizados usando el programa Statistical Package for the Social Sciences [SPSS] (Erazo, 2021). Entendiendo además que los estudiantes son legalmente dependientes de sus representantes y no pueden celebrar ningún acuerdo ni firmar ningún documento a título personal se contó con la aprobación de sus tutores legales quienes mediante su firma en una carta de autorización socializada y firmada entre las partes actuantes concedieron el permiso para la aplicación de los test en los cuales se basa el estudio.

\section{RESULTADOS}

Usando las pruebas de normalidad Shapiro-Wilk, considerando que la población es menor a 50 individuos se determinó que todas la variables en análisis cumplen con la condición de normalidad considerando una significancia de 0,000 para todas lo que indica que son paramétricas y con una confiablidad del 95\% para el análisis, así mismo para probar la hipótesis propuesta se utilizó la prueba T de Student relacionando las variables más significativas que permitieron certificar el impacto que tuvo la utilización de Genially con un $p$-valor de a $\leq 0,05$ para considerar a la hipótesis como valedera o el $p$-valor de $a \geq 0,05$ para la hipótesis nula 
Revista Interdisciplinaria de Humanidades, Educación, Ciencia y Tecnología

Año VII. Vol. VII. N³. Edición Especial III. 2021

Hecho el depósito de ley: pp201602FA4721

ISSN-L: 2542-3029; ISSN: 2610-802X

Universidad Nacional Experimental Francisco de Miranda (UNEFM). Santa Ana de Coro. Venezuela

Daniel Patricio Castro-Salinas; Sergio Constantino Ochoa-Encalada

Tabla 2.

Resultados generales del pre y post test con variables no asociadas.

\begin{tabular}{lccccccccccc}
\hline & \multicolumn{2}{c}{$\begin{array}{c}c \\
\text { V1 }\end{array}$} & \multicolumn{2}{c}{ V3 } & \multicolumn{2}{c}{ V6 } & \multicolumn{2}{c}{ V7 } & \multicolumn{2}{c}{ V9 } \\
& Pre & Post & Pre & Post & Pre & Post & Pre & Post & Pre & Post \\
\hline N & 13 & 13 & 13 & 13 & 13 & 13 & 13 & 13 & 13 & 13 \\
Sig. Bilateral & \multicolumn{2}{c}{0,067} & \multicolumn{2}{c}{0,067} & \multicolumn{2}{c}{0,070} & \multicolumn{1}{c}{1,000} & 0,116 \\
Media & 0,62 & 0,92 & 0,62 & 0,92 & 0,77 & 1,00 & 0,77 & 0,77 & 0,46 & 0,77 \\
Desviación & 0,506 & 0,277 & 0,506 & 0,277 & 0,439 & 0,000 & 0,439 & 0,439 & 0,519 & 0,439 \\
estandar & & & & & & & & & & \\
Media de error & 0,140 & 0,077 & 0,140 & 0,077 & 0,122 & 0,000 & 0,122 & 0,122 & 0,144 & 0,122 \\
estandar & & & & & & & & & & \\
\hline
\end{tabular}

Fuente: Encuesta.

Leyenda:

V1: Conceptos básicos de la célula

V3: Clasificación de organsimos vivos de acuerdo a su número de células

V6: Componentes principales de una célula eucariota

V7: Reconocimiento de las caracteristicas y funcionamiento del núcleo celular

V9: Reconocimiento de las caracteristicas y funcionamiento de la menbrana celular

En la tabla 2 se puede identificar que en las 5 variables consideradas como no asociadas los alumnos han demostrado un ligero incremento en el rendimiento de sus conocimientos con una diferencia de 0,30 en referencia al concepto básico de la célula y la clasificación celular de acuerdo al número de células, 0,33 en la identificación de los componentes básicos de una célula eucariota; no hay diferencia en reconocer las características y funcionamiento del núcleo y 0,31 puntos de diferencia en la caracterización de la membrana celular por lo que se asume que tanto el uso de recursos tradicionales e innovadores al momento de transmitir conocimientos surten efectos similares en los alumnos pero que también dejan abierta la posibilidad de mejorar o usar 
de manera diferente recursos que permitan innovar la forma de solventar el proceso de enseñanza aprendizaje.

Tabla 3.

Resultados generales del pre y post test con variables asociadas.

\begin{tabular}{lccccccccccc}
\hline & \multicolumn{2}{c}{ V2 } & \multicolumn{2}{c}{ V4 } & \multicolumn{2}{c}{ V5 } & \multicolumn{2}{c}{ V8 } & \multicolumn{2}{c}{ V10 } \\
& Pre & Post & \multicolumn{2}{c}{ Pre } & Post & Pre & Post & Pre & Post & Pre & Post \\
\hline N & 13 & 13 & 13 & 13 & 13 & 13 & 13 & 13 & 13 & 13 \\
Sig. Bilateral & \multicolumn{2}{c}{0,300} & \multicolumn{2}{c}{0,003} & \multicolumn{2}{c}{0,009} & \multicolumn{2}{c}{0,41} & & 0,004 \\
Media & 0,69 & 1,00 & 0,38 & 0,92 & 0,46 & 0,92 & 0,46 & 0,85 & 0,31 & 0,85 \\
$\begin{array}{l}\text { Desviación } \\
\text { estandar }\end{array}$ & 0,480 & 0,000 & 0,506 & 0,277 & 0,519 & 0,277 & 0,519 & 0,376 & 0,480 & 0,376 \\
$\begin{array}{l}\text { Media de error } \\
\text { estandar }\end{array}$ & 0,133 & 0,000 & 0,140 & 0,077 & 0,144 & 0,077 & 0,144 & 0,104 & 0,133 & 0,104 \\
\hline
\end{tabular}

Fuente: Encuesta.

Leyenda:

V2: Clasificación celular considerando su estructura

V4: Identificación de características propias de las células procariotas

V5: Identificación de características propias de las células eucariotas

V8: Reconocimiento de las características y funcionamiento del citoplasma celular

V10: Reconocimiento de las características y funcionamiento de la pared celular

A diferencia de los resultados expuesto en la tabla 2, en la tabla 3 se verifican mayores diferencias en las medias aritméticas entre las variables considerando que los estudiantes obtuvieron una diferencia de 0,31 en referencia a identificar a las células mediante su estructura; 0,54 y 0,46 en cuanto a la identificación de características de células procariotas y eucariotas; 0,39 y 0,50 en el reconocimiento y funcionamiento del citoplasma y pared celular respectivamente, facultando mencionar que los estudiantes mejoraron su rendimiento notablemente después de la aplicación de la estrategia de 
innovación para el caso sugiriendo hacer uso de nuevas formas de propagación de conocimientos y evaluación de resultados.

Tabla 4.

Comparación de promedios obtenidos con la ayuda de recursos tradicionales (Pre test) y con el uso de Genially (post test).

\begin{tabular}{ccccccc}
\hline & Test & N & Media & $\begin{array}{c}\text { Desviación } \\
\text { estandar }\end{array}$ & $\begin{array}{c}\text { Media de } \\
\text { error estandar }\end{array}$ & $\begin{array}{c}\text { Significancia } \\
\text { (bilateral) }\end{array}$ \\
\hline \multirow{2}{*}{ Promedio } & Recursos tradicionales & 13 & 6,46 & 3,205 & 0,889 & 0,002 \\
& Recursos innovadores & 13 & 9,69 & 0,855 & 0,237 & \\
\hline
\end{tabular}

Fuente: Encuesta.

En la tabla 4 se puede evidenciar que luego haber hecho uso de recursos tradicionales para la explicación y aplicado el pretest se obtuvo como media sobre diez puntos posibles una calificación de 6,46 que en comparación con la escala cuantitativa y cualitativa del Ministerio de Educación del Ecuador está considerada como próximo a alcanzar los aprendizajes requeridos, a diferencia del promedio obtenido en el post test con el uso de recursos innovadores en donde la media es de 9,69 indicando que dominan los aprendizajes requeridos, con una significancia bilateral de 0,002, con una variación entre medias de 3,23 puntos demostrando un evidente incremento en los resultados obtenidos luego de la aplicación de la estrategia generando mayor interactividad, comprensión del tema y retención de información facultando la utilización de recursos que busquen la innovación en la educación y que sean aplicables en el proceso de interaprendizaje. 


\section{PROPUESTA}

La aplicación de estrategias innovadoras en la educación permite obtener mejores resultados en la evaluación de conocimientos en los estudiantes y el uso de Genially facilita por tanto se propone el siguiente esquema de aplicación que consta de 4 etapas: Instruir, planificar, aplicar y evaluar con miras a mejorar el rendimiento de los estudiantes en el dominio científico que abarca el área de ciencias naturales.

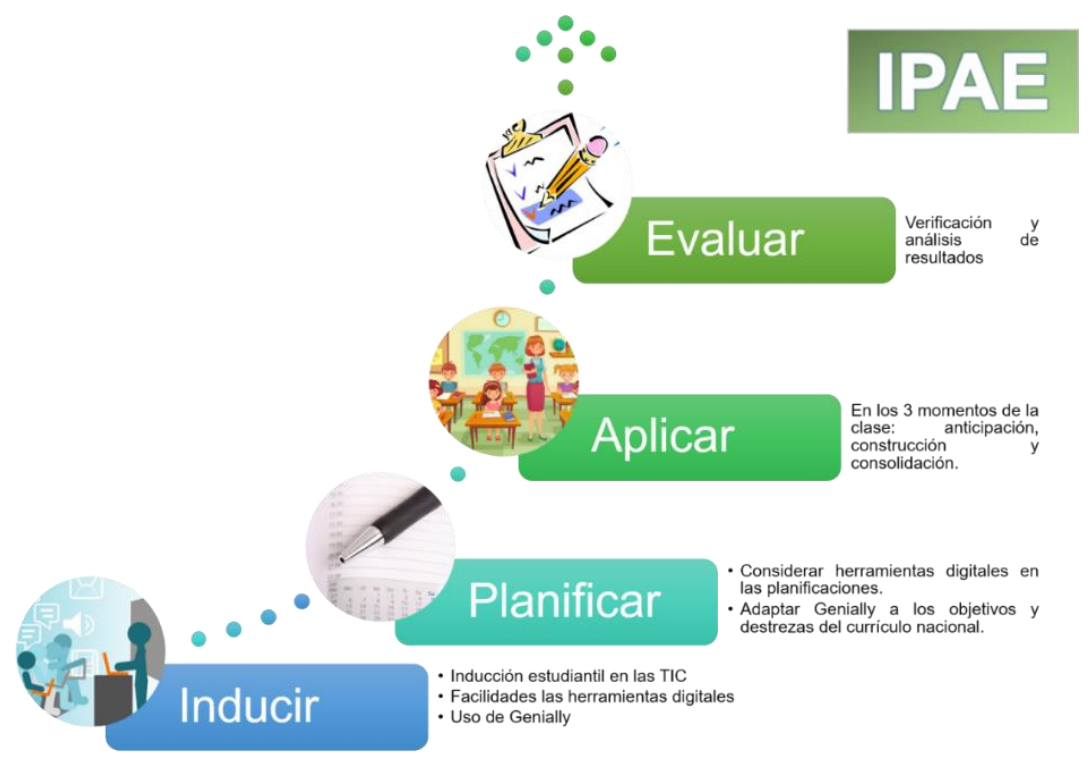

Figura 1. Esquema de implementación de la herramienta digital Genially en el proceso de interaprendizaje de ciencias naturales.

Fuente: Los autores.

Inducir: Motivar a los estudiantes a cerca de la importancia del uso de las tecnologías de la información y la comunicación en el proceso de interaprendizaje y por ende de las herramientas digitales disponibles entre ellas Genially recalcando en este proceso las ventajas que supone su uso en comparación con las maneras tradicionales de impartir conocimientos, explicando de manera y concisa su funcionamiento, los usos que se podrían dar y la adaptabilidad que presenta la herramienta. 
Planificar: Se consideró la aplicación de la herramienta Genially observando los lineamientos emitidos por el MINEDUC en los cuales se establece a las TIC como un eje transversal en el proceso educativo en todas las áreas por lo que haciendo uso de la destreza pertinente se procedió a elaborar una presentación en la herramienta mencionada destacando los tres momentos de la clase: anticipación, construcción y consolidación de los conocimientos, enfocando toda la planificación acorde al objetivo establecido para la clase.

Aplicar: Teniendo elaborada la planificación realizada se procede a dar la clase haciendo uso de Genially mediante la presentación creada para el efecto comenzando con la clase con la anticipación realizando preguntas como ¿Qué conoce usted de la célula? ¿Por qué a la célula se la considera como la base esencial de la vida? ¿Por qué los animales son diferentes a las platas? ¿Las células de las bacterias y los seres humanos son iguales? Entre otras, posterior a eso en la construcción de los conocimientos se procede a hacer uso de textos cortos, fotografías y videos para la explicación de conceptos generales de célula, clasificaciones por morfología y número, las semejanzas y diferencias de cada clasificación y la desagregación en características y funcionalidad de cada una de las partes de una célula eucariota de manera participativa mediante una investigación previa realizada por los alumnos que unificaron y reafirmaron criterios durante la clase, finalmente para la consolidación de aprendizajes se usó la misma herramienta y a través del juego de preguntas catalogado como "Quizz Marcianitos" propio de la aplicación y modificado con preguntas acordes al tema tratado durante la clase.

Evaluar: Para la evaluación se aplicó una evaluación en línea a través de Microsoft Forms, la cual mediante diez preguntas de opción múltiple con una valoración de un punto por pregunta permitió definir mediante la media aritmética comparada con la evaluación antecedente a la rendida después de haber aplicado la herramienta y comparar si ha surtido efecto o no en la comprensión, retención y transmisión de conocimientos por parte de los alumnos evaluados. 


\section{CONCLUSIONES}

La motivación a través de estrategias y herramientas que permiten la Gamificación y la interacción de textos y recursos audiovisuales en el proceso de interaprendizaje es esencial considerando que los alumnos motivados son los que obtienen mejores resultados al momento de evaluar los nuevos conocimientos ya que el alumno considera al aprendizaje como un reto que debe superar a través de los niveles que vaya pasando recordando lo aprendido y mejorando en lo que presente dificultades con el fin de obtener mejores resultados y evitar que el "juego" no sea superado buscando la forma de llegar a la meta de forma individual o colaborativa.

El uso de las TIC en la educación es fundamental en un mundo globalizado y por ende hace necesaria la capacitación y el entendimiento del funcionamiento de plataformas y herramientas digitales que posibilitan y facilitan la labor docente entendiendo y reconociendo que la forma de transmitir conocimientos ha ido cambiando con el pasar de los años y que la tecnología usada de manera adecuada puede ser una gran aliada en la educación y puede mejorar el rendimiento académico de los educandos.

La aplicación de Genially durante la clase favorece la comprensión, asimilación y retención de conocimientos en los alumnos debido a que, al permitir mayor interacción, participación y Gamificación crea un ambiente propicio e idóneo para la transmisión de conocimientos debido a que los alumnos sienten mayor motivación e interés por aprender situación que se evidencia con la comparación de los promedios obtenidos en las dos evaluaciones aplicadas.

\section{REFERENCIAS}

Arias-Gallegos, W. L. (2015). Tecnologías de la información y la comunicación en colegios públicos y privados de Arequipa. [Information and communication technologies in public and private schools of Arequipa]. Interacciones, 1(1), 11-28. https://doi.org/10.24016/2015.v1n1.1 
Asamblea Nacional Constituyente. (2008). Constitución de la República del Ecuador. [Constitution of the Republic of Ecuador]. Asamblea Constituyente. https://doi.org/10.2307/j.ctvm204k6.6

Bieliková, V. (2019). Aplikácia niektorých prvkov gamifikácie a prevráteného učenia v triede ELE. [Aplicación de algunos elementos de la gamificación y del aprendizaje invertido en el aula de ELE]. [MASARYKOVA UNIVERZITA]. In Magisterská diplomová práce (Issue 1). https://n9.cl/xnja6

Byrd-Orozco, A. (2015). La docencia en el Siglo XXI: antecedentes, contexto y posibilidades. [Teaching in the 21st Century: background, context and possibilities]. Razón y Palabra, 20(92), 1412-1424. https://n9.cl/0boqv

Contreras-Espinosa, R. S. (2016). Juegos digitales y gamificación aplicados en el ámbito de la educación. [Digital games and gamification applied in the field of education]. RIED. Revista Iberoamericana de Educación a Distancia, 19(2), 27-33. https://n9.cl/puy2b

Covarrubias-Hernández, L. Y. (2021). Educación a distancia: transformación de los aprendizajes. [Distance education: learning transformation]. Telos Revista de Estudios Interdisciplinarios En Ciencias Sociales, 23(1), 150-160. https://doi.org/10.36390/telos231.12

Diario Córdoba S.A.U. (2015). Genially una herramienta que pretende revolucionar la comunicación y la educación. https://n9.cl/2tp9f

Erazo Álvarez, J. C. (2021). Capital intelectual y gestión de innovación: Pequeñas y medianas empresas de cuero y calzado en Tungurahua-Ecuador. Revista De Ciencias Sociales, 27, 230-245. Recuperado a partir de https://produccioncientificaluz.org/index.php/rcs/article/view/37004

Espejo, R., \& Sarmiento, R. (2017). Metodologías activas para el aprendizaje: manual de apoyo docente. [Active methodologies for learning: a teacher's support manual]. In Universidad Central de Chile. https://n9.cl/zs5t

García-Aretio, L. (2020). COVID-19 y educación a distancia digital: preconfinamiento, confinamiento y posconfinamiento. [COVID-19 and digital distance education: preconfinement, confinement and postconfinement]. RIED. Revista Iberoamericana de Educación a Distancia, 24(1), 1-25. https://doi.org/10.5944/ried.24.1.28080 
García-Lázaro, I. (2019). Escape Room como propuesta de gamificación en educación. [Room Scape as a proposal for gamification in education]. Revista Educativa Hekademos, 27, 71-79. https://n9.cl/nhbwl

Hart, J. (2021, September 1). Top tools for learning 2021. [Las mejores herramientas para aprender 2021]. Results of the 15th Annual Survey. https://www.toptools4learning.com/

Hepp-K, P., Prats-Fernández, M. Á., \& Holgado-García, J. (2015). Formación de educadores: la tecnología al servicio del desarrollo de un perfil profesional innovador $y$ reflexivo. [Educator training: technology at the service of the development of an innovative and reflective professional profile]. RUSC. Universities and Knowledge Society Journal, 12(2), 30-42. https://n9.cl/ek8dj

Hermosa Del Vasto, P. M. (2015). Influencia de las tecnologías de información y comunicación (TIC) en el proceso enseñanza-aprendizaje: una mejora de las competencias digitales. [Influence of information and communication technologies (ICT) in the teaching-learning process: an improvemen. Revista Científica General José María Córdova, 13(16), 121-132. https://n9.cl/4ut82

Hernández-Sampieri, R., Fernández-Collado, C., \& Baptista-Lucio, M. del P. (2014). Metodología de la investigación. [Research Methodology]. https://n9.cl/2i4

Hernández-Sampieri, R., \& Mendoza-Torres, C. P. (2018). Metodología de la investigación: Las rutas de la investigación. [Research methodology: The routes of research]. In Metodología de la investigación. Las rutas cuantitativa, cualitativa y mixta. https://n9.cl/br1sy

Holguín-García, F. Y., Holguín-Rangel, E. G., \& García-Mera, N. A. (2020). Gamificación en la enseñanza de las matemáticas: una revisión sistemática. [Gamification in mathematics education: a systematic review]. Telos, 22(1), 61-71. https://n9.cl/5zmgg

Instituto Nacional de Evaluación Educativa [INEVAL]. (2019). Informe de resultados "Ser Bachiller" año lectivo 2018-2019. [Report of results "Ser Bachiller" school year 20182019]. https://n9.cl/jd76f 
Llerena-Medina, E. G., \& Rodríguez-Hurtado, C. P. (2017). Kahoot! A Digital Tool for Learning Vocabulary in a language classroom. [iKahoot! Una herramienta digital para aprender vocabulario en el aula de idiomas]. Revista Publicando, 4(12), 441-449. https://n9.cl/h4yv3x

Macías-Espinales, A. (2018). Gamificación en el desarrollo de la competencia matemática: plantear y resolver problemas. [Gamification in the development of mathematical competence: pose and solve problems]. SINAPSIS, 1(12), 1-18. https://n9.cl//49ax9

Mendoza-Castillo, L. (2020). Lo que la pandemia nos enseñó sobre la educación a distancia. [What the pandemic taught us about distance education]. Revista Latinoamericana de Estudios Educativos, 50(ESPECIAL), 343-352. https://doi.org/10.48102/rlee.2020.50.especial.119

Ministerio de Educación del Ecuador [MINEDUC]. (2011). Ley Organica de Educacion Intercultural. [Organic Law of Intercultural Education]. Boletín Oficial Del Estado. https://n9.cl/en2uy

Ministerio de Educación del Ecuador [MINEDUC]. (2015). Reglamento general a la Ley Orgánica de Educación Intercultural. [General Regulations to the Organic Law of Intercultural Education]. Dirección Nacional de Normativa Jurídico Educativa. https://n9.cl/bca5

Ministerio de Educación del Ecuador [MINEDUC]. (2016). Currículo [Curriculum]. https://educacion.gob.ec/curriculo/

Nikoletta-Zampeta, L., Nannan, X., Juho, H., Kostas, K., \& Vassilios, A. (2020). The effect of challenge-based gamification on learning: An experiment in the context of statistics education. [El efecto de la gamificación basada en retos sobre el aprendizaje: Un experimento en el contexto de la enseñanza de la estadística]. International Journal of Human-Computer Studies, 144(November 2019), 1-14. https://doi.org/10.1016/j.ijhcs.2020.102496

Organización de las Naciones Unidas para la Educación, la C. y la C. [UNESCO]. (2017). E2030: Educación y habilidades para el siglo XXI. [E2030: Education and Skills for the 21st Century]. In Oficina Regional de Educación para América Latina y el Caribe. https://n9.cl/hkrg 
Organzación de las Naciones Unidas. (2020). Informe de políticas: La educación durante la COVID-19 y después de ella. [Policy Brief: Education during COVID-19 and beyond.]. In Informe de políticas:la educación durante la COVID - 19 y después de ella (Vol. 1, Issue 1). https://n9.cl/cyde

Ortiz-Colón, A. M., Jordán, J., \& Agredal, M. (2018). Gamificación en educación: una panorámica sobre el estado de la cuestión. [Gamification in education: an overview of the state of the art]. Educação e Pesquisa, 44(0), 1-17. https://n9.cl/89aha

Ospina-Rodríguez, J. (2006). La motivación, motor del aprendizaje. [Motivation, the engine of learning]. Revista Ciencias de La Salud, 4(Esp), 158-160. https://n9.cl/naye

Palazón-Herrera, J. (2015). Motivación del alumnado de educación secundaria a través del uso de insignias digitales [Motivation of secondary education students through the use of digital badges]. Opción, 31(1), 1059-1079. https://n9.cl/o6gi

Pulido-Polo, M. (2015). Ceremonial y protocolo: métodos y técnicas de investigación científica. [Ceremonial and protocol: methods and techniques of scientific research]. Opción, 31(1), 1137-1156. https://n9.cl/91rf8

Ramos-Galarza, C. A. (2020). Los alcances de una investigación. [The scope of an investigation]. CienciAmérica, 9(3), 1-5. https://doi.org/10.33210/ca.v9i3.336

Real-Pérez, M., Sánchez-Oliva, D., \& Padilla-Model. (2021). Proyecto África «La Leyenda de Faro»: Efectos de una metodología basada en la gamificación sobre la motivación situacional respecto al contenido de expresión corporal en educación secundaria. [Africa Project «La Leyenda de Faro»: Effects of a methodology . Retos, 42, 567574. https://doi.org/10.47197/retos.v42i0.86124

Rodríguez, F., \& Campión, R. S. (2015). Gamificación: Como motivar a tu alumnado y mejorar el clima en el aula. [Gamification: How to motivate your students and improve the classroom climate]. In Editorial Océano S.L.U. https://doi.org/10.21556/edutec.2016.55.705

Sánchez-Peris, F. J. (2015). Gamificación. [Gamification]. Education in the Knowledge Society, 16(2), 13-15. https://n9.cl/lchvi

Sellan-Naula, M. E. (2017). Importancia de la motivación en el aprendizaje. [Importance of motivation in learning]. Revista Electrónica Sinergias Educativas, 2(1), 1-3. https://doi.org/10.31876/s.e.v2i1.20 
Serna-Huesca, O., Sánchez-Serrano, C. J., \& Rubio Martínez, I. (2015). Los nuevos retos y demandas de la educación "Desarrollo de competencias". [The new challenges and demands of education "Competence development"]. Atenas, 3(31), 1-7.

Sierra-Villamil, G. M. (2016). Liderazgo educativo en el siglo XXI, desde la perspectiva del emprendimiento sostenible. [Educational leadership in the 21 st century, from the perspective of sustainable entrepreneurship]. Revista EAN, 81, 111-128. https://doi.org/10.21158/01208160.n81.2016.1562

Silva-Quiroz, J., \& Maturana-Castillo, D. (2017). Una propuesta de modelo para introducir metodologías activas en educación superior. [A proposed model for introducing active methodologies in higher education]. Innovación Educativa, 17(73), 117-131. https://n9.cl/tjvw

Valenzuela, J., Muñoz-Valenzuela, C., Silva-Peña, I., Gómez-Nocetti, V., \& PrechGandarillas, A. (2015). Motivación escolar: Claves para la formación motivacional de futuros docentes. [School motivation: Keys to future teachers' motivational training]. Estudios Pedagogicos, 41(1), 351-361. https://n9.cl/7hkna

Villalustre-Martínez, L., \& Del Moral-Pérez, M. E. (2015). Gamificación: Estrategia para optimizar el proceso de aprendizaje y la adquisición de competencias en contextos universitarios. Digital Education Review, 27, 13-31. https://n9.cl/e0kvy

Zepeda-Hernández, S., Abascal-Mena, R., \& López-Ornelas, E. (2016). Integración de gamificación y aprendizaje activo en el aula. [Integration of gamification and active learning in the classroom]. Ra Ximhai, 12(6), 315-326. https://doi.org/10.35197/rx.12.01.e3.2016.21.sz 